\title{
Jefferson.
}

\section{Trans-sacrococcygeal Joint Ganglion Impar Injection for Treatment of Pudendal Neuralgia (3 cases report)}

\section{Matthew McAuliffe, MD1; Linqiu Zhou, MD2}

'Department of Rehabilitation Medicine, Thomas Jefferson University Hospital, Philadelphia, PA

${ }^{2}$ Department of Rehabilitation Medicine, Jefferson Medical College, Thomas Jefferson University, Philadelphia, PA

\section{PROGRAM DESCRIPTION}

\section{HISTORY}

Three patients, 2 male and 1 female were diagnosed with pudendal neuralgia. The female patient developed pudendal neuralgia secondary to post-herpetic neuralgia. The two male patients were caused from likely repetitive sitting injury. One patient was a truck driver causing him to sit for long periods of time and the other patien was a professional writer. The three patient initially all attempted conservative therapies, however their pain persisted. They elected to undergo Trans-sacrococcygeal joint ganglion impar injection for treatment of pudendal neuralgia,

\section{ANATOMY}

The sacral sympathetic trunk as it runs through the pelvis sits anterior to the sacrum, medial to the anterior sacral foramina. As the trunks travel distally they converge and end in front of the sacralcoccygeal joint in what is known as the ganglion impar or ganglion of Walther. The ganglion impar is in the midline and is the distal termination of the sympathetic chains. It may lie up to $1.9 \mathrm{~cm}$ away termination of the sympathetic chains. It may lie up to $1.9 \mathrm{~cm}$ away fromi it's classically described position and may lie as inferior as the midpoint of the coccyx. ${ }^{1,2}$

\section{PROCEDURE}

All of them underwent series of ganglion impar injection through the sacrococcygeal joint under IV sedation. After a 25 -gauge spinal needle was passed the sacrococcygeal joint 2-3 millimeters and negative aspiration of blood, $0.5 \mathrm{cc}$ of contrast dye was injected to confirm the appreciate needle positioning. Then, $5 \mathrm{cc}$ mixtures of steroid and Marcaine were injected weekly for three. The repeat injections were offered, if pain relief was not satisfied.

\section{RESULTS}

The symptoms of female patient with Post herpetic neuralgia completely resolved after having three series of injections with three years of follow-up care.

The male patients received greater than $75 \%$ of pain reduction with the same treatment. The male patients were both able to decrease their chronic pain medications as well as return to their respective jobs with some on the job modifications such as using a cushion seat.

The outcome measures evaluated were overall pain level, functional improvements, and opioid medication usage. There was significant improvement in each case regards to the above outcome measures.
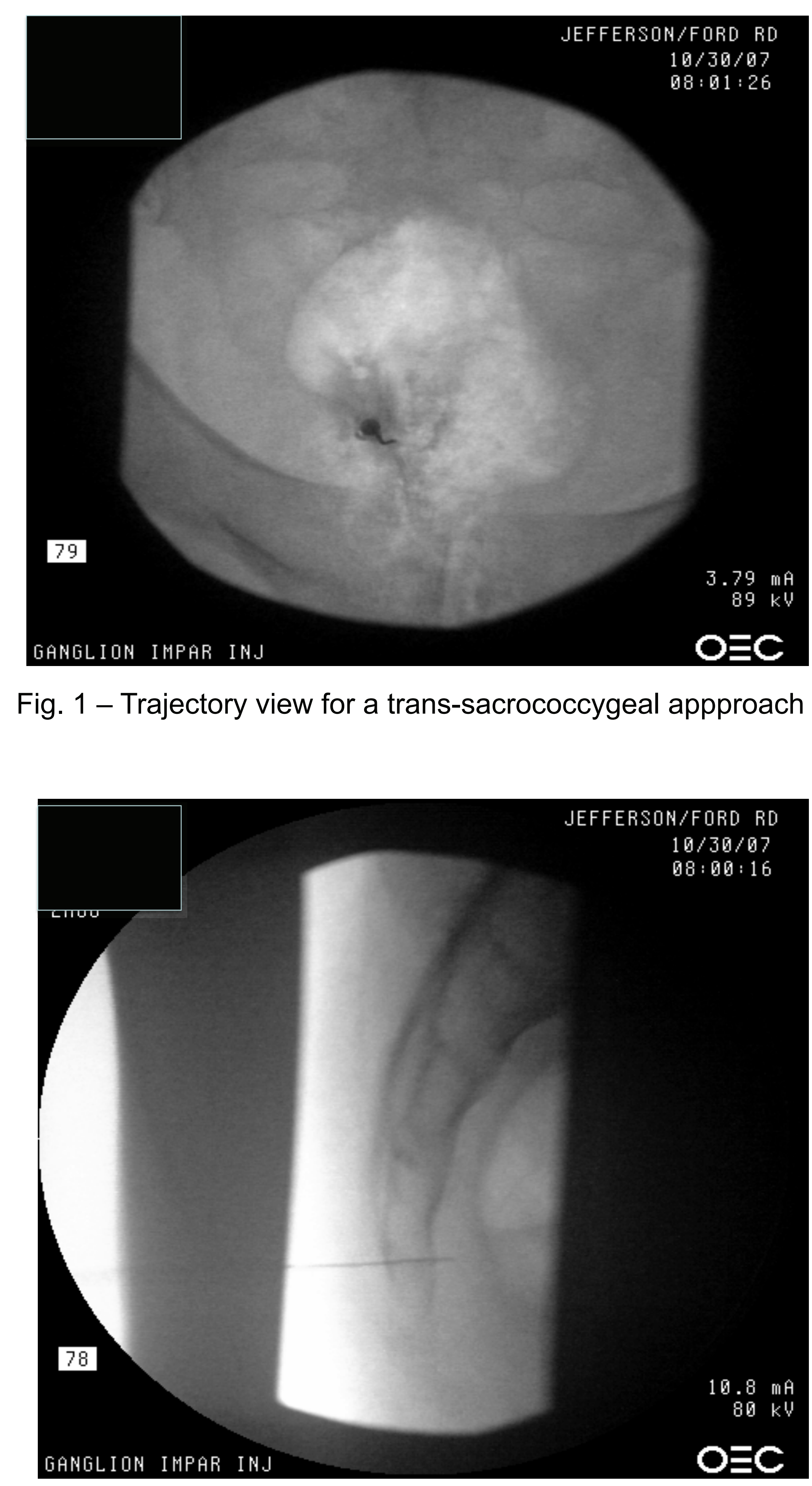

Fig. 2 - Lateral view for trans-sacrococcygeal approach with needle in place

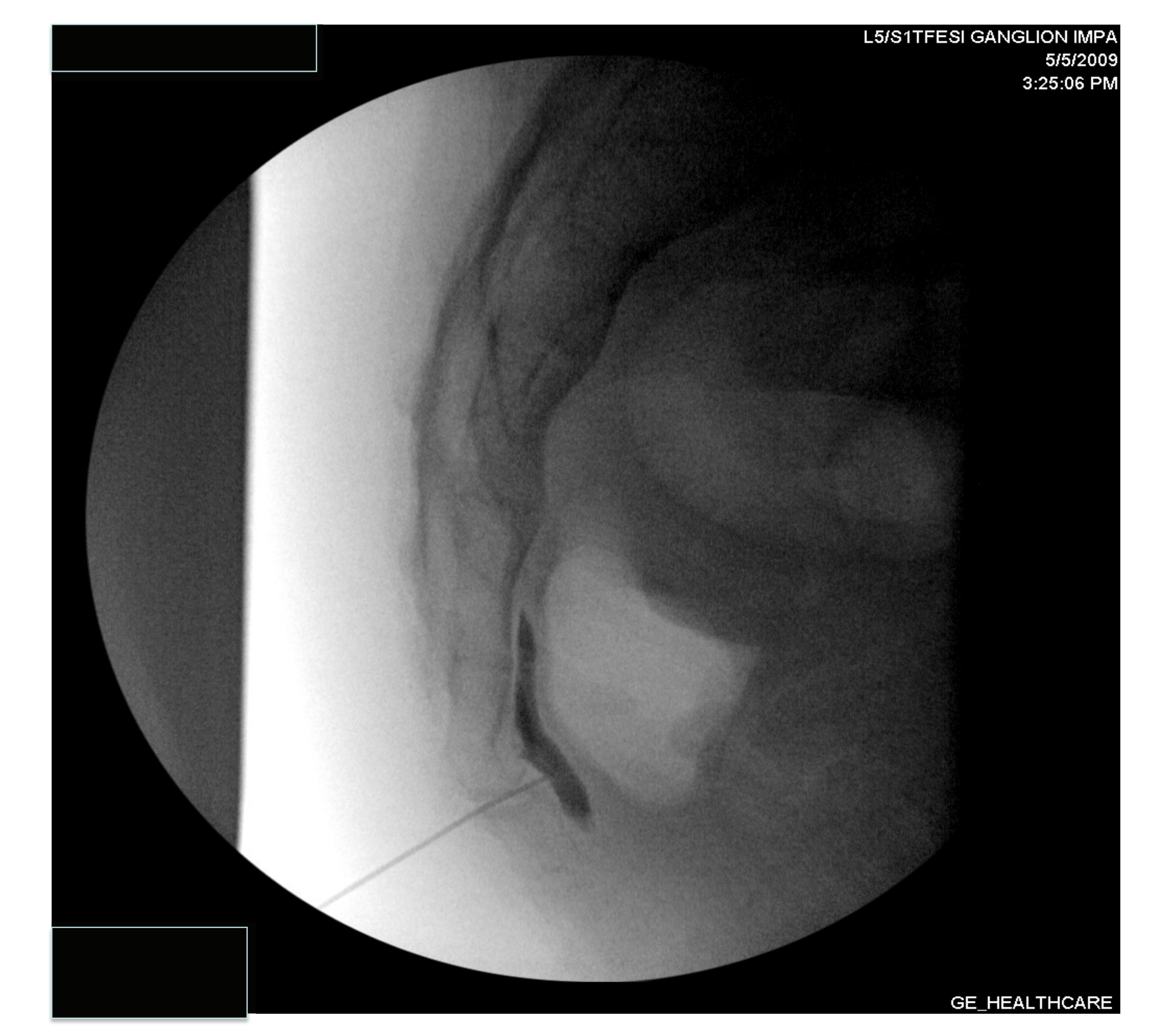

Fig. 3 - Lateral view cofirming neele position with injection of dye

\section{DISCUSSION}

The case report describes the efficacy of trans-sacrococcygeal joint impar injection for treatment of pudendal neuralgia in a select number of cases. Other treatment options for this condition include physical therapy such as pelvic floor stretching exercises. The importance of good ergnomics and sitting positions should be emphasized. Oral analgesics are commonly used to help with pain. For refractory pudendal neuralgia there are case reports of cryoablation and thermal coagulation with radiofreqency ablation. ${ }^{1,3,4}$

The ganglion impar can be approached by injection in a number of different ways including though the sacrococcygeal joint. Alternative approaches include through the anococcygeal ligament either via a midline or paramedian approach or through the intercoccygeal joint space. Approaches through the anococcygeal ligament are thought to have a greater risk of rectal perforation.

In each case described here where a trans-sacrococcygeal appproach was used, there was evidence for significant improvement in pain in the three patients who underwent the procedures, an increase in their functional capacity, and in these particularly motivated patients a decrease in the amount of chronic pain medications they used. Transsacrococcygeal joint ganglion impar injection provides an alternative to more invasive interventions, as well as some non-interventional methods of treating chronic pain that may not be as effective.

\section{CONCLUSION}

The pathophysiology of pudendal neuralgia is not precisely known but likely can be caused from multiple different kinds of etiologies. The treatment for these patients has generally been challenging often without satisfactory results. On the other hand, ganglion impar injection through sacrococcygeal joint is a simple, relatively painless and safe procedure. Our cases have demonstrated that series of ganglion impar injections are effective for treatment of pudendal neuralgia.

\section{REFERENCES}

Scott-Warren JT, Hill V, Rajasekaran A. Ganglion Impar Blockade: A review. Curr Pain (2013) 17:306.

2. Oh CS, Chung IH, Ji HJ, et al. Clinical imp

3. Loev MA, Varklet VL, Wilsey BL, et al. Cryoabl

4. Karaman $\mathrm{H}$, Tüfek A, Kavak GO, et al.Would pulsed radiofrequency applied to different 2011:61:879-85. 